\title{
Radiative and convective heat transfer in microelectronics
}

\author{
by B. Więcek ${ }^{1}$ H. Madura ${ }^{2}$
}

${ }^{1}$ Institute of Electronics, Technical University of Łodž, 18-22 Stefanowskiego str. 90-924 Łódž, Poland,

${ }^{2}$ Institute of Optoelectronics, Military Academy of Technology, 2 Kaliski str. 01-489 Warsaw, Poland

\begin{abstract}
This paper presents numerical and experimental results of heat transfer by radiation, convection and conduction in hybrid microelectronic circuits. We chose a heat source with a non-uniform temperature distribution, which agrees with typical cases frequently met in electronics. In this work we evaluate a complex heat transfer coefficient including the non-linear phenomenon for radiative and convective heat dissipation. We apply thermography to confirm the correctness of the simulation.
\end{abstract}

\section{Introduction}

It has already been underlined by many authors that in order to evaluate more precisely the temperature in microelectronic devices, one should include convection and radiation into the modelling of heat transfer [1-5]. In many previous works, convection was simply approximated by transfer coefficient $\alpha$, taken from the tables and being typically constant over the temperature range. Because of a multidimensional nature of convection and the non-linear characteristics for both the convection and the radiation, there are not many works which include these phenomenon into the entire heat removal modelling until now $[1,4]$. In this work we model conduction together with radiation and convection, applying their non-linear parameters. Also we include the temperature-dependent emissivity for metals. Simulations yield non-uniform temperature distributions in the electronic device, and using these distributions we evaluate the non-linear complex heat transfer coefficient, comparing it to published and measured data.

\section{The non-linear heat transfer model for conduction, radiation and convection}

In this work we present a numerical model of heat removal for a hybrid long resistor placed on a ceramic substrate (Fig.1). The problem can be reduced to a one-dimensional modelling Because of $h_{s} / h_{r} \gg>1$ and $h_{s} / t_{s}<<1$, where $h_{r}, h_{s}$ and $t_{s}$ are the resistor height, substrate height and thickness of the substrate, respectively. This is because of the symmetrical heat transfer in the substrate in the vertical direction, neglecting the fact that the upper side of the substrate is cooled down worse, because of warm fluid moving up [5].

The model starts from the energy conservation law expressed by heat fluxes:

$$
-S_{s} d y \frac{d \varphi_{s}}{d y}=2 S_{a}\left(\varphi_{r}+\varphi_{c}\right)
$$

where: $\varphi_{s}, \varphi_{n} \varphi_{c}$ denote the heat fluxes that correspond to conduction in the substrate, radiation and convection from the source to the ambient. $S_{s}$ and $S_{a}$ denote the areas that the fluxes go through, as shown in Fig.2. The fluxes are described as follows:

$$
\begin{aligned}
\varphi_{s} & =-\lambda_{s} \frac{d T}{d y} \\
\varphi_{c} & =\alpha_{c}\left(T-T_{a}\right) \\
\varphi_{r} & =\varepsilon \sigma\left(T^{4}-T_{a}^{4}\right)
\end{aligned}
$$

where: $\alpha_{c}$ - convective heat transfer coefficient, $\varepsilon$ - emissivity, $\sigma$ - Boltzman constant, $\lambda_{s}$ substrate thermal conductivity and $T_{a}$ - ambient temperature. 


\section{http://dx.doi.org/10.21611/qirt.1996.010}

In our approach we assume a non-linear convective heat transfer coefficient, defined by (3).

$$
\alpha_{c}=\alpha_{0}\left(\frac{\Delta T}{\Delta T_{0}}\right)^{\frac{1}{n}}
$$

where $\alpha_{0}$ denote the transfer coefficient corresponding to temperature difference $\Delta T_{o}$, e.g.: $20^{\circ} \mathrm{C}$, $\Delta T=T-T_{\mathrm{a}}$. Parameter $n$ can vary in the range 4-10 [4-5]. In practice, $n=10$ denotes that transfer coefficient doubles when the temperature difference rises 10 times, what takes place for nonuniform temperature distribution on the substrate. The better cooling conditions are for $n=4 \$ / 4$ Law), but it is only valid for constant source temperature, and is very rarely met in practice. Moreover, $n=4$ has been derived from a simplified 1-D natural convection model, and has not been confirmed by the 2-D convection simulations [4].

Finally, the non-linear energy equation that has to be solved takes the following form:

$$
t_{s} \lambda_{s} \frac{d^{2} T}{d y^{2}}=2 \varepsilon \sigma\left(T^{4}-T_{a}^{4}\right)+\frac{2 \alpha_{0}}{\sqrt[n]{\Delta T_{0}}}\left(T-T_{a}\right)^{\frac{1+n}{n}}
$$

where: $t_{s}$ is the tickness of a substrate.

The boundary conditions for our problem are:

$$
\left.\frac{d T}{d y}\right|_{y=0}=\frac{P_{z}}{2 \lambda_{s} t_{s}} \text { and }\left.\frac{d T}{d y}\right|_{y=h_{s}}=0 \text { where: } P_{z}=P_{z}\left[\frac{W}{m}\right]
$$

Equation (4) can be solved by Runge-Kutta numerical approach, yielding the temperature distribution on the substrate, and a number of additional data, such as the conjugate heat transfer coefficient for convection and radiation.

\section{Simulation results for black and grey bodies}

The level of the power delivered to the heat source has an influence on the maximum and mean temperature in the substrate. The relation $T_{\max }$ versus power is non-linear because of the non-linear coefficients presented above. In this work we found the influence of the power dissipated in the substrate on the temperature and the transfer coefficient.

The transfer coefficient $\alpha$ is defined in various ways, but for this work we propose the following definitions:

$$
\alpha_{T_{\max }}=\frac{P_{z}}{2 h_{s}\left(T_{\max }-T_{u}\right)} \quad \alpha_{\tau_{\text {seat }}}=\frac{P_{z}}{2 h_{s}\left(T_{\text {meam }}-T_{u t}\right)}
$$

The first simulations have been performed for black body $(\varepsilon=1)$ and for $n=4(1 / 4$ Law $)$ for a convective heat transfer coefficient given by (3). The parameters of the ceramic substrate and the air are presumed to be independent on the temperature and are as follows: $T_{a}=300 \mathrm{~K}$, $\lambda_{s}=20 \mathrm{~W} / \mathrm{Km}, t_{s}=0.4 \mathrm{~mm}, h_{s}=3 \mathrm{~cm}$. The parameters of the non-linear model of convection according to the $1 / 4$ Law are: $\alpha_{0}=7 \mathrm{~W} / \mathrm{m}^{2}, \Delta \mathrm{T}_{0}=20^{\circ} \mathrm{C}$

The results presented in Fig.4-5 confirm the high contribution of radiation in the total heat dissipation, especially for $T_{\max }>450 \mathrm{~K}$. Above this temperature the heat is more easily removed by radiation than convection.

Using the results of the simulation we can approximate by exponential functions both the power dissipated in the substrate and the total transfer coefficient versus temperature: $P_{z}=f\left(T_{\max }\right)$ and $\alpha=f\left(T_{\max }\right)$ as:

$$
P_{z}=P_{0}\left(\frac{\Delta T_{\max }}{\Delta T_{0}}\right)^{m} \quad P_{z}=P_{0}\left(\frac{\Delta T_{\text {mean }}}{\Delta T_{0}}\right)^{m} \quad \alpha_{\text {max }}=\alpha_{0}\left(\frac{\Delta T_{\text {max }}}{\Delta T_{0}}\right)^{m} \quad \alpha_{\text {mean }}=\alpha_{0}\left(\frac{\Delta T_{\text {mean }}}{\Delta T_{0}}\right)^{m}
$$

The results for black body ( $\varepsilon=1)$ using the $1 / 4$ Law for convection model are in Table 1. 
Table 1. $P_{\mathrm{z}}=f\left(T_{\max }\right)$ and $\alpha_{\mathrm{z}}=f\left(T_{\max }\right)$ approximation parameters

\begin{tabular}{|c|c|c|c|c|c|c|}
\cline { 2 - 7 } \multicolumn{1}{c|}{} & \multicolumn{2}{c|}{ Convection } & \multicolumn{2}{c|}{ Radiation } & \multicolumn{2}{c|}{ Convection +Radiation } \\
\cline { 2 - 7 } \multicolumn{1}{c|}{} & $\mathrm{P}_{\mathrm{o}} / \alpha_{0}$ & $\mathrm{~m}$ & $\mathrm{P}_{\mathrm{o}} / \alpha_{0}$ & $\mathrm{~m}$. & $\mathrm{P}_{\mathrm{o}} / \alpha_{0}$ & $\mathrm{~m}$ \\
\hline $\mathrm{P}_{\mathrm{z}}\left(\mathrm{T}_{\text {max }}\right)$ & 7.41 & 1.20 & 4.68 & 1.44 & 11.98 & 1.23 \\
\hline $\mathrm{P}_{\mathrm{z}}\left(\mathrm{T}_{\text {mean }}\right)$ & 8.42 & 1.25 & 4.76 & 1.58 & 15.21 & 1.30 \\
\hline$\alpha_{\text {max }}$ & 6.34 & 0.21 & 4.50 & 0.43 & 10.86 & 0.23 \\
\hline$\alpha_{\text {mean }}$ & 6.85 & 0.26 & 4.64 & 0.49 & 12.87 & 0.27 \\
\hline
\end{tabular}

From Table 1, we can come to some general conclusions:

- Radiation and convection remove a comparable amount of heat in the temperature range accepted in microelectronics (e.g.: below $200^{\circ} \mathrm{C}$ )

- The functions $P_{z}=f\left(T_{\max }\right)$ and $\alpha=f\left(T_{\max }\right)$ are non-linear, and as far as the power dissipated by convection and radiation are comparable, radiation-convection and convection models give very similar variation of the $T_{\max }$ and $\alpha$ over temperature (parameter $m$ does not change very much). However the levels of energy dissipated are quite different

- From $T_{\text {max }}=150^{\circ} \mathrm{C}$ radiation dominates over convection

As we have already found, the convection coefficient does not increase so fast as the $1 / 4$ Law predicts, especially for heat sources with non-uniform temperature distribution [4]. The better approximation for transfer heat coefficient is obtained for $1 / n=0.1-0.17(3)$.

The next simulations were performed for grey bodies and for $1 / n=0.125$. Now the parameters of the non-linear model of convection are: $\alpha_{0}=5.5 \mathrm{~W} / \mathrm{m}^{2}$ for $\nabla \mathrm{T}_{0}=20^{\circ} \mathrm{C}[4-5]$.

Table 2. $P_{z}=f\left(T_{\max }\right)$ for gray bodies

\begin{tabular}{|c|c|c|c|c|c|c|}
\hline \multicolumn{7}{|c|}{$T_{\max }[\mathrm{K}]$} \\
\hline $\mathrm{P}_{2}[\mathrm{~W} / \mathrm{m}]$. & $\varepsilon=0$ & $\varepsilon=0.2$ & $\varepsilon=0.4$ & $\varepsilon=0.6$ & $\varepsilon=0.8$ & $\varepsilon=1$ \\
\hline 25 & 398 & 375 & 363 & 354 & 348 & 343 \\
\hline 50 & 482 & 434 & 412 & 397 & 387 & 379 \\
\hline 75 & 563 & 486 & 454 & 434 & 420 & 410 \\
\hline 100 & 640 & 532 & 492 & 467 & 451 & 438 \\
\hline 125 & 716 & 573 & 526 & 498 & 478 & 464 \\
\hline 150 & 790 & 611 & 557 & 525 & 504 & 488 \\
\hline
\end{tabular}

The general conclusion drawn from the simulations for grey bodies is that,

- The decay of $T_{\max }$ is the largest $(70 \%)$ for $\varepsilon=0-0.4$, which is the case in the practice (Table 2, Fig.5).

The approximation $P_{z} \sim \Delta T^{m}{ }_{T \max }$ for e.g. $\varepsilon=0.4$ gives unexpected result - factor $m$ is quite high indicating the high significance of radiation in heat removal.

$$
P_{z}=P_{0}\left(\frac{\Delta T_{\max }}{\Delta T_{0}}\right)^{m} \text { where: }\left\{\begin{array}{l}
P_{0}=5.11, m=1.32 \text { for } \varepsilon=0.4 \\
P_{0}=8.72, m=1.27 \text { for } \varepsilon=1.0
\end{array}\right.
$$

The materials used in microelectronics e.g.: metals was taken into consideration for modelling, as well. The total, normal emissivity $\varepsilon_{T, n}$ for metals strongly depends upon the temperature, giving the next nonlinear phenomenon included in this work $[1,2,3]$.

$$
\begin{aligned}
& \varepsilon_{T, n}=0.576 \sqrt{\rho(T) T}-0.124 \rho(T) T \\
& \rho(T)=\rho_{273} \frac{T}{273}
\end{aligned}
$$

where $\rho(T)$ denotes the electrical resistivity of a metal. Applying the total emissivity of metal as $\varepsilon_{T} \approx 1.2 \varepsilon_{T, n}$ [2], the energy equation (4) can be extended using (9), giving the results presented in Fig. 6 and Table 3. 
Table 3. $P_{\mathbf{z}}=f\left(T_{\max }\right)$ for metals

\begin{tabular}{|c|c|c|c|c|}
\hline \multicolumn{5}{|c|}{$\mathrm{T}_{\max }[\mathrm{K}]$} \\
\hline $\mathrm{P}_{\mathrm{z}}[\mathrm{W} / \mathrm{m}]$. & Al. & $\mathrm{Fe}$ & $\mathrm{Fe}-42 \mathrm{Ni}^{*}$ & $\varepsilon=0$ \\
\hline 25 & 394 & 391 & 383 & 398 \\
\hline 50 & 472 & 466 & 477 & 482 \\
\hline 75 & 543 & 531 & 501 & 563 \\
\hline 100 & 607 & 588 & 547 & 640 \\
\hline
\end{tabular}

\section{Experiments}

We measured the temperature distribution on a ceramic substrate with a long resistor on it (Fig. 7a), using thermographic system TVS4000-HUGHES, supported with a computer interface for capturing the images and powerful software running under WIN'95. For the measurement we used a hybrid resistors circuit, placed nearly in the middle of the substrate as shown in Fig. 7. Both sides of the substrate were covered by thin coating with emissivity $\varepsilon \approx 0.9$. The measurements were very close to the modelling, as presented in (10).

$$
P_{z}=P_{0}\left(\frac{\Delta T_{\max }}{\Delta T_{0}}\right)^{m} \quad \text { where: } P_{0}=0.69, m=1.22
$$

From simulations we obtained $m=1.26-1.27$ for $\varepsilon=0.8-1$, while the thermography measurements gave $m=1.22$.

\section{Conclusions}

The simulations and the experiments presented in this paper yield with some remarkable conclusions. First of all, the radiation and convection remove a comparable amount of heat in the temperature range of working microelectronics devices (e.g.: below $200^{\circ} \mathrm{C}$ ). $P_{z}=f\left(T_{\text {max }}\right)$ and $\alpha=f\left(T_{\max }\right)$ are non-linear. For comparable power dissipated by convection and radiation, the radiation-convection and the convection models give a similar variation of the $T_{\max }$ and $\alpha$ over the used temperature range ( $m$ parameter does not change very much). $T_{\max }=150^{\circ} \mathrm{C}$ is the point where radiation begins to dominate over the convection for the heat sources with nonuniform temperature distribution. Low emissivity reduces the radiative energy dissipated significantly, although the decay of $T_{\max }$ is at largest $(70 \%)$ for $\varepsilon=0-0.4$. We got a very good agreement between the thermography measurements and the model results.

\section{Acknowledgments}

The authors thank Prof. G. De Mey from the University of Gent (ELIS), Belgium for his helpful scientific suggestions and inspirations used in this work.

\section{REFERENCES}

[1] Siegel (R.) and Howell (J).- Thermal radiation heat transfer. New York, Hemisphere Publishing Corp, 1989.

[2] Burakowski (T.), Giziński (J.) and Sala (A.). - Promienniki podczerwieni, Warsaw, WNT, 1970 [in Polish].

[3] Sala (A.) - Radiacyjna wymiana ciepla. Warsaw, WNT, 1982 [in Polish]

[4] Więcek (B.), De Mey (G.). Evaluation of of heat dissipation by convection for VLSI circuits. Proc. TERMINIC'96 Conf., Budapest, Sept.25-27,1996, p143-148

[5] Więcek (B.). Quantitative approach into the heat transfer by convection in microelectronics with thermography measurements. Proc. QIRT'96. Stuttgart, Sept. 2-5, 1996 


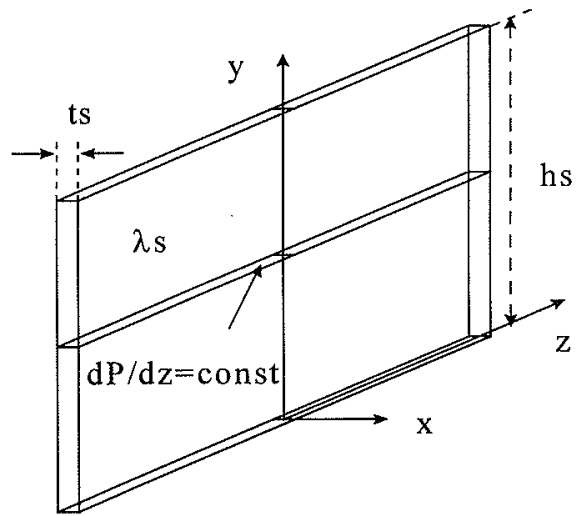

Fig.1. Substrate with the heat source

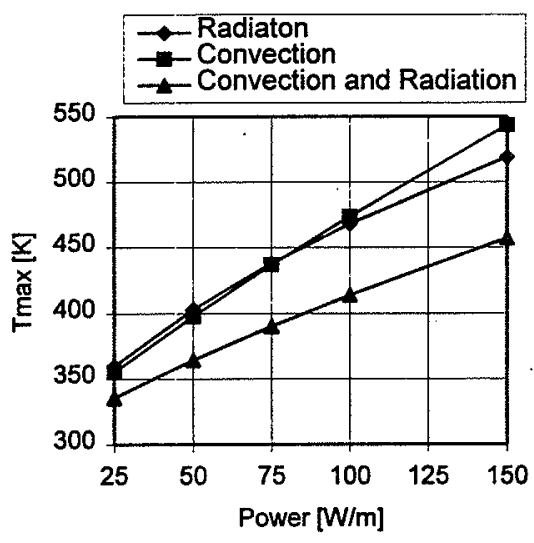

Fig. 3. $T_{\max }$ versus power

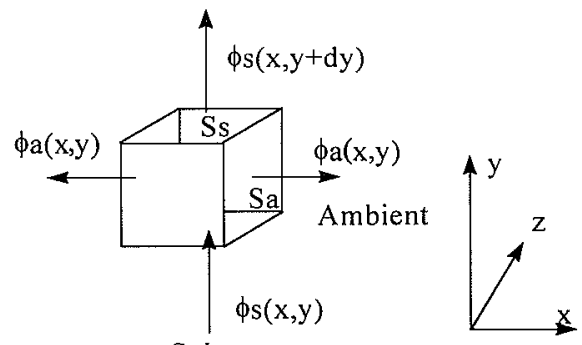

Substrate

Fig.2. Heat fluxes in the substrate

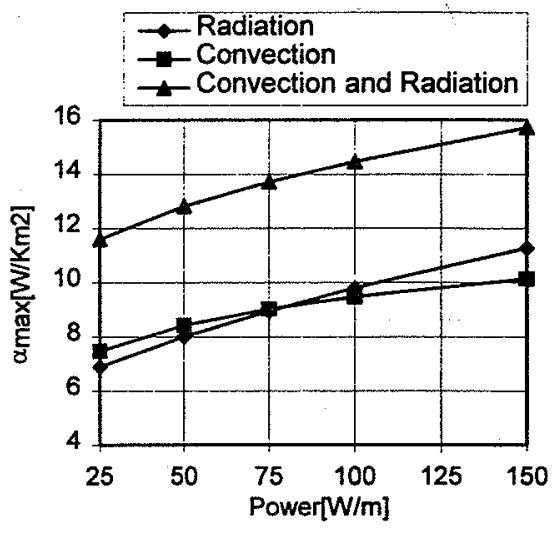

Fig. 4. $\alpha_{\max }$ versus power 


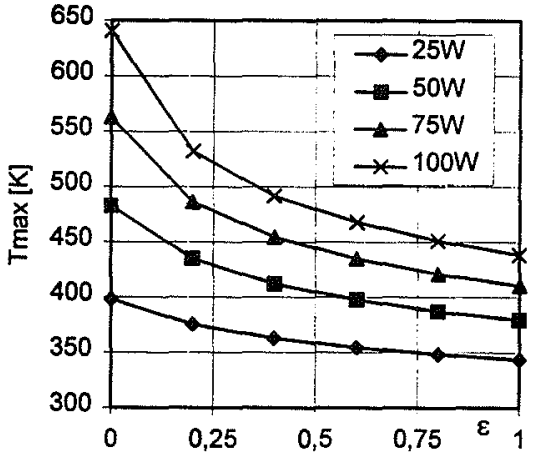

Fig. 5. $T_{\max }=f(\varepsilon)$ for gray bodies
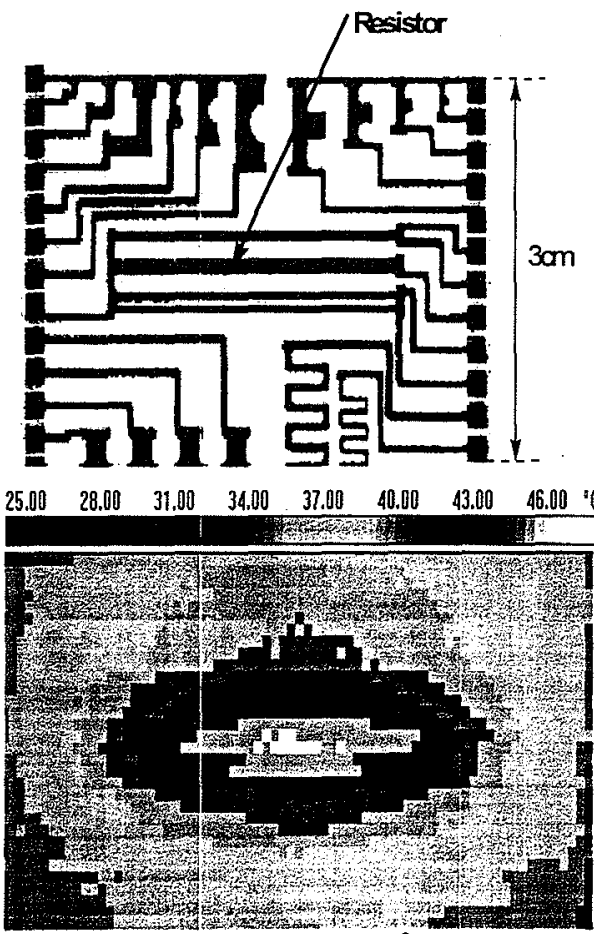

$\mathrm{P}_{\mathrm{z}} / \mathrm{P}_{0}=2, \nabla \mathrm{T}_{\max }=27^{\circ} \mathrm{C}$

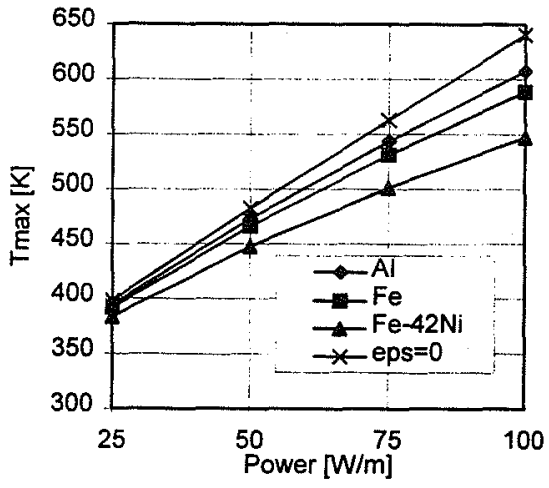

Fig. 6. $T_{\max }=f\left(P_{z}\right)$ for metals compared to the convective heat dissipation only $(\varepsilon=0)$
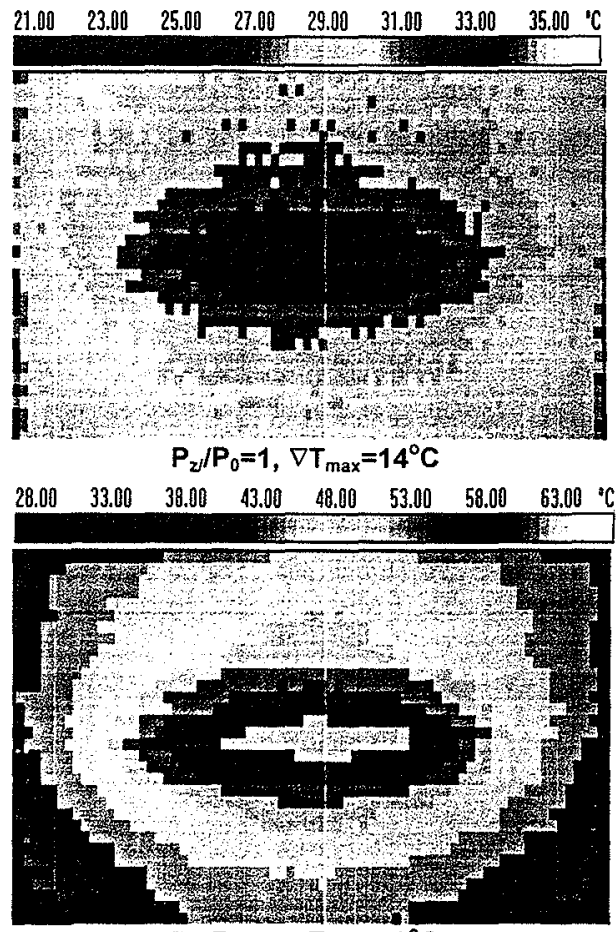

$\mathrm{P}_{\mathrm{z}} / \mathrm{P}_{0}=4, \nabla \mathrm{T}_{\max }=43^{\circ} \mathrm{C}$

Fig. 7. Resistor on the ceramic substrate and temperature distribution for various power 